\title{
Plantas medicinales usadas en la terapéutica de pacientes con hipertensión y diabetes mellitus tipo II, de la región indígena Tepehuana
}

\author{
Medicinal Plants Used in the Therapeutics of Patients with Hypertension and Type II Diabetes, \\ of the Tepehuana Indigenous Region
}

\author{
Azucena E Jiménez-Corona ${ }^{a}$, Isaac Ayipey Mateo-Rivera ${ }^{b}$
}

\begin{abstract}
:
This study was realized utilizing the northeastern medicinal plant collection from the state of Veracruz (aka the Tepehuana region) with the aim of raising awareness of their physiologically-active compounds and their therapeutic application in type 2 diabetes and hypertension. These plants were collected in their wild type state and extensive research of their known therapeutic effects as alternative allopathic treatment in patients suffering the mentioned chronic degenerative diseases was conducted. Mexican herbalism is an extensive field and its origins date back to ancient times when our ancestors began the arduous task of classifying medicinal plants according to their observed effects; however, over time the knowledge and use of these plants has greatly diminished, replaced by commercially-available drugs and the pharmaceutical industry. Furthermore, the great biodiversity of plants still conserved in modern-day Mexico continues to contribute to allopathic treatments in the population, particularly in the rural areas. Therefore, our main objective is to disclose novel physiologically-active compounds from these plants, with the purpose amplifying their therapeutic targets and reducing the adverse effects that synthetic drugs exert on vital organs, including the liver and kidneys. The future perspectives of this study focus on the extraction of these compounds, and their pharmacological and toxicological characterization, employing both cellular and animal models, with the goal of bench-side to bed-side applicability.
\end{abstract}

Keywords:

Diabetes, Hypertension, Plantago major, Cecropia obtusifolia, Guazuma ulmifolia, Solanum nigrum. Tepehuana, medicinal plants

\section{Resumen:}

Este estudio fue desarrollado primeramente realizando la recolección de plantas de uso medicinal, desde su lugar de origen en la parte Noreste del estado de Veracruz (Tepehuana). Con la finalidad de dar a conocer la importancia de los principios activos que contienen estas plantas y su uso terapéutico para la prevención de enfermedades cardiovasculares, diabetes tipo II e hipertensión. Estas plantas se recolectaron en su estado silvestre y de esta manera llevar a cabo una búsqueda exhaustiva del efecto terapéutico como una alternativa en el tratamiento alopático en pacientes que padecen estas enfermedades crónicas degenerativas. La herbolaria mexicana tiene una extensa riqueza, desde tiempos remotos nuestros ancestros han clasificado las plantas medicinales a partir de su efecto, sin embargo, con el tiempo se está perdiendo el conocimiento y uso de estas plantas. No obstante, hoy en día en México conservamos una gran biodiversidad en plantas, que coadyuvan en los tratamientos alopáticos utilizados en el tratamiento de diversas patologías. Por lo tanto, nuestro objetivo principal es dar a conocer nuevos principios activos provenientes de estas plantas, con el propósito, de tener una gama más amplia en los tratamientos terapéuticos que disminuyan los efectos adversos que provocan los fármacos sintéticos y dañan gravemente órganos vitales como hígado y riñones. Las perspectivas de este estudio se enfocan en la extracción de los principios activos de estas plantas y el desarrollo de nuevos experimentos farmacológicos y toxicológicos, a nivel celular y en modelos animales, para que posteriormente podamos trasladar su uso en pacientes que padezcan estas enfermedades.

\section{Palabras Clave:}

Diabetes, Hipertensión, Plantago major, Cecropia obtusifolia, Guazuma ulmifolia, Solanum nigrum. Tepehuana, Plantas medicinales

\footnotetext{
a Autor de Correspondencia: Azucena Eunice Jiménez Corona. Profesor investigador de la Escuela Superior de Huejutla, Universidad Autónoma del Estado de Hidalgo Email: azucena_jimenez@uaeh.edu.mx

b Isaac Ayipey Mateo Rivera. Alumno de la licenciatura Medico Cirujano de la Escuela Superior de Huejutla, Universidad Autónoma del 


\section{Introducción}

Uno de los objetivos principales de este estudio es dar a conocer la extensa riqueza que poseen los pueblos indígenas en su territorio geográfico, una pequeña pero significativa parte de la biodiversidad de plantas medicinales que existen en el mundo, en este caso particular el pueblo Tepehua. Desde tiempos milenarios sabemos que, la medicina herbolaria se transmite de generación en generación, sin embargo, hoy en día se ha ido perdiendo el conocimiento y el uso de estas plantas medicinales. Podríamos hacer referencia que, los abuelos de estos pueblos indígenas trataban a sus enfermos no solo con el uso de las plantas medicinales, si no también hacían uso de los cinco elementos principales ya conocidos. Comprendiéndose así, a la enfermedad como un castigo por desobedecer a los dioses. Asimismo, hacían uso de distintas partes y sustancias que extraían de diversos animales.

La diabetes mellitus es una enfermedad metabólica y crónica degenerativa, asociada al desarrollo de una serie de diversas complicaciones irreversibles, causando a su vez invalidez en los pacientes y una afectación en la calidad de vida. Muchos de los pacientes al ser diagnosticados como diabéticos se aterrorizan, debido a que la calidad de vida disminuye considerablemente, puesto que estos pacientes estarán dependiendo de distintos tratamientos y fármacos para controlar los niveles de glucosa y en diversas ocasiones de tensión arterial, lo que dependerá de un buen apego al tratamiento y a la dieta, puesto que, de lo contrario se desencadenarán diversas complicaciones que podrán llevar a una alta mortalidad. [1]

Se define a la diabetes como una enfermedad que tiene su origen por diversas causas, se caracteriza por hiperglucemia secundaria a deficiencia de acción o cantidad de insulina, que si no se trata de manera apropiada puede provocar alteraciones metabólicas agudas y trastornos crónicos que deterioran la función y la estructura de diversos órganos. [2]

En la antigüedad las poblaciones desconocían el uso de fármacos para tratar distintas patologías, como es el caso de la diabetes, incluso desconocían a la misma patología. En la actualidad existen poblaciones que acostumbran el uso de remedios herbolarios para tratar diversos signos y síntomas, relacionados al síndrome metabólico, que en este caso es la diabetes y la hipertensión arterial. Asimismo, los habitantes de la etnia Tepehua de la comunidad de Tierra Colorada pertenecientes al municipio Tlachichilco de la zona Norte del estado de Veracruz, aún conocen una extensa variedad de plantas que ellos usan para tratar o controlar los distintos síntomas de la diabetes e hipertensión arterial.
El botánico Ángel Beyra, realizó un estudio de las plantas medicinales más utilizadas por la población cubana, realizó una evaluación con métodos científicos para poder observar sus efectos farmacológicos y toxicológicos que le permitieron establecer a lo que hoy se le conoce como medicina alopática (moderna), ya que la mayoría de la medicina herbolaria ha demostrado una gran efectividad, ya que los efectos secundarios son mínimos o no los producen, por lo cual se han usado de generación en generación ganando prestigio en la práctica médica actual. [3]

El Dr. Mankil Juong y colaboradores, realizaron investigaciones sobre los efectos hipoglucémicos de algunas plantas usadas como remedios antidiabéticos, mediante estos estudios se proporcionó información sustentada a las poblaciones rurales que las usan, y posteriormente se inició una investigación para poder saber los mecanismos de la actividad hipoglucémica de estas plantas. Un ejemplo claro de ellos es Pterocarpus marsupium, los componentes de su corteza tienen un gran efecto hipoglucémico, el extracto significativamente redujo los niveles de azúcar en la sangre de $72.32 \%$ de los pacientes $2 \mathrm{~h}$ después de la administración oral, 21 días después de la administración oral se observó que aumentaba el filtrado glomerular, además, el extracto también mostró un efecto favorable en disposición de la glucosa en las ratas hiperglucémicas alimentadas con glucosa, actuando de manera constante sobre los transportadores GLUT. Asimismo, encontraron que en las especies de la planta Talinum paniculatum Gaertner tienen como principio activo al hexa-acetato de javaberina A y hexa-acetato de javaberina $B$ que son inhibidores de la producción de TNF-a por macrófagos y células grasas, por lo que concluyeron que T. paniculatum es muy útil como suplemento dietético y para la prevención de la diabetes. [4]

Por otro lado, Dr. Santosh realizó estudios sobre la planta Cynodon dactylon, describiendo su gran importancia como diurético, permitiendo el aumento del filtrado glomerular y manteniendo normales los niveles de glucosa. A su vez, describieron que estos tratamientos son efectivos, ya que los efectos secundarios que producen son mínimos o no los producen. Por lo que estos tratamientos homeopáticos, son de bajo costo comparados con los agentes hipoglucémicos sintéticos orales. [5]

Por lo tanto, es de gran importancia dar a conocer el efecto farmacológico y toxicológico de los extractos provenientes de estas plantas e implementarlos en la terapéutica de pacientes con diabetes mellitus tipo II e hipertensión arterial. Por lo que, caracterizar los principios activos de estas plantas y dar a conocer la dosis y efecto de estos productos naturales provenientes de éste y otros pueblos indígenas, sería de gran impacto en salud pública, ya que 
traería consigo un gran avance en el desarrollo y aplicación de nuevos fármacos de bajo costo; lo cual sería un gran apoyo para la población en general. Mencionado lo anterior, el primer objetivo de este estudio en un futuro a corto plazo será la extracción y caracterización de los principios activos de estas plantas medicinales, mediante el desarrollo de experimentos farmacológicos y toxicológicos en cultivos celulares y modelos experimentales en animales.

\section{PLANTAS USADAS POR LA REGIÓN:}

\section{PLANTAGO MAJOR}

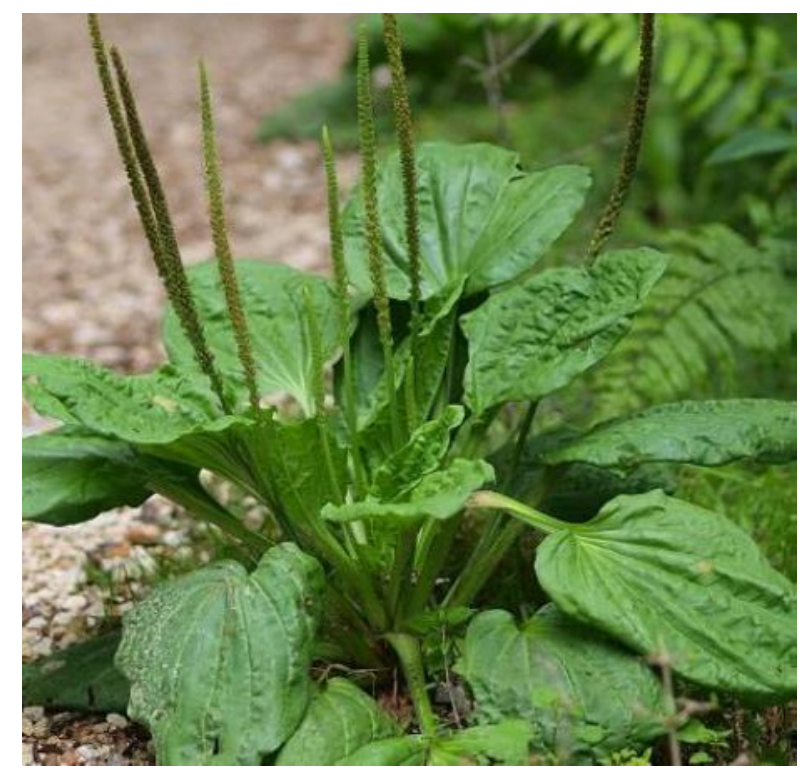

Figura1. Nombre común: Llantén. Nombre científico: Plantago major. Orden: Lámiales. Familia: Plantaginaceae. Género: Plantago

Uso medicinal: Ayuda a tratar infecciones urinarias, tratar heridas, interrumpe hemorragias, alivia los dolores de úlceras y de hemorroides, antiséptico, diurético, antiinflamatorio, ayuda a mantener estables los niveles de hipertensión, regula la glucosa en la sangre. Suele usarse en infusiones.

Los estudios más recientes han mostrado que $P$. major es usada en muchas partes del mundo, en el tratamiento de una serie de enfermedades principalmente aquellas que ayudan a tratar alergias dermatológicas, y enfermedades infecciosas, de igual manera aquellos problemas relacionados con los órganos digestivos y respiratorios, a su vez, se ha reportado su uso para mantener una buena circulación sanguínea, logrando aliviar el dolor y a disminuir la fiebre. Las investigaciones de la Dra. Anne Berit Samuelsen, se basaron en el estudio de las semillas de esta planta, teniendo como resultados, que estas tienen un alto contenido de proteínas y algunos carbohidratos como: glucosa, fructosa, xilosa y ramnosa, así como la sacarosa disacárida y el trisacárido plantaose. Constituido de un $61 \%$ xilosa, $13.2 \%$ de arabinosa y $24 \%$ de ácido galacturónico. Asimismo, realizaron estudios realizando la extracción de sus principios activos mediante ebullición, de la cual se obtuvo un $78 \%$ de xilosa, $13.2 \%$ de arabinosa, $3 \%$ de galactosa y $6.2 \%$ de ácido galacturónico. [6]

Entre las investigaciones de la Dra. Samuelsen, reportaron que $\mathrm{P}$. major presenta un alto contenido de ácido cafeico, que es un gran antioxidante que ayuda en la prevención del cáncer. Teniendo un papel directamente en la disminución de la síntesis y unión de radicales libres a las moléculas del ADN. En forma paralela, también se ha demostrado que un alto co

nsumo de antioxidantes ayuda a disminuir de manera progresiva el estrés oxidativo y por lo tanto, una disminución de la tensión arterial.

Por otro lado, en pueblos indígenas de Argentina se ha implementado desde hace varias décadas el uso del $P$. major para tratar mialgias y odontalgias, además en el tratamiento de regulación de los niveles de glucosa. [7] Es importante destacar que, en la comunidad indígena Tepehuana de Tierra Colorada, Tlachichilco del Estado de Veracruz, han usado esta planta para tratar y llevar un control de los niveles elevados de glucosa, pero ésta no es la única planta usada, a su vez se realizan infusiones de otras plantas que se describen a continuación.

\section{CECROPIA OBTUSIFOLIA}

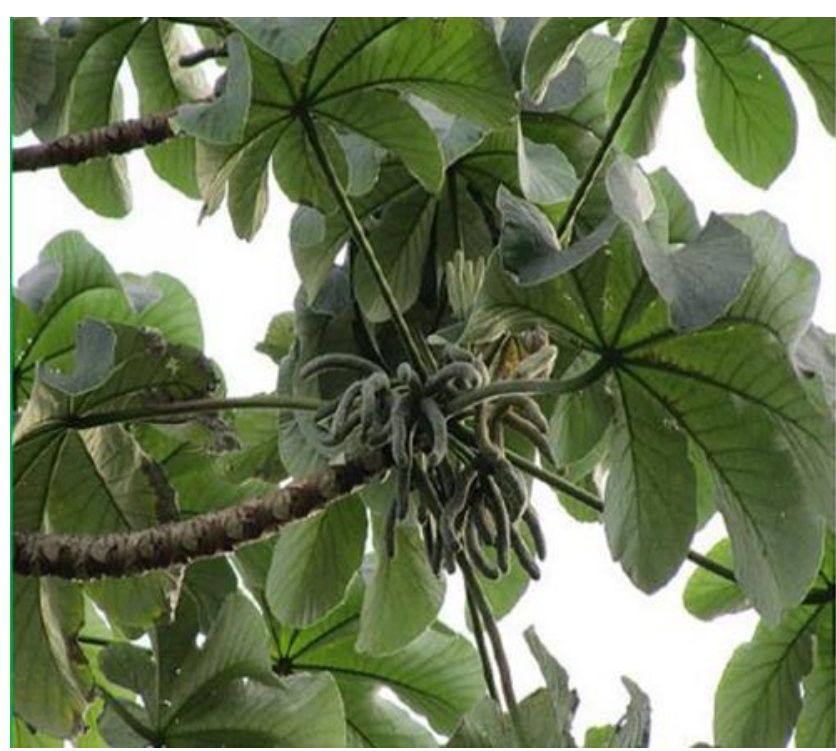


Figura 2. Nombre común: Hormiguillo, Nombre científico: Cecropia obtusifolia, Nombre en Tepehua: Aqo, Orden: Rosales, Familia: Urticaceae, Género: Cecropia.

Uso medicinal: Utilizada comúnmente para mantener estables los niveles de glucosa, purifica los riñones y ayuda a evitar problemas cardiacos y a que acelera el metabolismo de los lípidos. Se prepara una infusión de estas hojas.

El extracto acuoso de Cecropia tiene un efecto hipoglucémico y su uso terapéutico fue descubierto en experimentaciones con ratas y conejos hiperglucémicos. [8]

Las decocciones de agua de 'Guarumbo' se usan tradicionalmente para el trateamiento de la diabetes mellitus tipo II, particularmente en los estados mexicanos de Oaxaca, Hidalgo y Veracruz. La planta se vende en varios mercados tradicionales sola o mezclada con otras plantas como complejo antidiabético. [9]

El objetivo del estudio realizado por la Dra. Cristina fue investigar el efecto hipoglucemiante de ésta y otros extractos de $\mathrm{C}$. obtusifolia en ratas diabéticas inducidas por estreptozotocina e identificar los principales constituyentes químicos en la planta. La diabetes fue inducida por un solo medicamento intraperitoneal, con una dosis estreptozotocina recién preparada (STZ) solución (Sigma, No. 242-646-8) (50 mg / kg en acetato tampón 0.1 $\mathrm{M}, \mathrm{pH} 4.5)$ a ratas en ayuno durante la noche. [8]

La diabetes se identificó por polidipsia, poliuria, midiendo los niveles de glucosa plasmática 48 horas después de la inyección de STZ (sin ayuno). Las ratas fueron divididas en grupos de seis, de los cuales cuatro grupos eran de once ratas y los dos restantes de siete y ocho ratas. Después de un tratamiento agudo con $\mathrm{C}$. obtusifolia, se confirmó su papel como un agente hipoglucemiante. [8]

En ratas diabéticas, tanto los extractos como los dos compuestos aislados mostraron efectos significativos de hipoglucemia. El extracto acuoso en dosis de $90 \mathrm{mg} / \mathrm{kg}$ de peso corporal mostró actividad a los 60 minutos, con una reducción significativa $(P \leq 0.001)$, después de 60 minutos, la importancia se redujo. Posteriormente, se observó el efecto máximo de los extractos de agua después de $180 \mathrm{~min}$. Por lo que, el extracto butanólico condujo a una disminución significativa en la concentración de glucosa plasmática en comparación con el control, en dosis de $9 \mathrm{mg} / \mathrm{kg}$ de peso corporal, el efecto fue significativo después de 60 min con $\mathrm{P} 0.005$ en curso con $(P \leq 0.001)$ a 120 y $180 \mathrm{~min}$. En dosis de $15 \mathrm{mg} / \mathrm{kg}$ de peso corporal, la actividad fue significativa desde 60 min hasta 180 min con $(P \leq 0.001)$. La actividad máxima se observó después de 180 min comparable al extracto de agua. El ácido clorogénico 1 y la isoorientina 2 mostraron una actividad similar con $(P \leq 0.001)$ a 60,120 y $180 \mathrm{~min}$. En forma paralela con el grupo tratado con glibenclamida (3 $\mathrm{mg} / \mathrm{kg}$ ), que a su vez produjo una disminución en comparación con los controles, con $(P \leq 0.001)$ a los 60 $\min$ y $180 \mathrm{~min}$. [8]

Usualmente, las personas del pueblo indígena Tepehuana beben la infusión de las hojas después de hervir entre tres y cinco hojas (aproximadamente $36 \mathrm{~g}$. a $60 \mathrm{~g}$.) en 1 litro de agua, posteriormente se ingiere durante todo el día en porciones pequeñas como agua de tiempo.

Más tarde, se probó en modelos con animales, realizando ahora un estudio en 12 pacientes diabéticos tipo 2 recientemente diagnosticados, controlados solo con dieta y ejercicio. Los niveles séricos de glucosa, colesterol, triglicéridos e insulina se determinaron cada 15 días; HbA1c, ALT, AST y ALKP se midieron cada mes. Se detectó una reducción significativa de la glucosa después de 4 semanas de administración. La HbA1c también se redujo significativamente después de 6 semanas de tratamiento. No hubo cambios significativos en el colesterol, triglicéridos ALT, AST, ALKP o insulina. Tampoco se observaron efectos colaterales. Posteriormente se suspendió la administración del extracto y al cabo de 34 semanas, las concentraciones de glucosa y $\mathrm{HbA} 1 \mathrm{c}$ aumentaron y alcanzaron niveles más altos que los basales. [8]

Podemos concluir que C. obtusifolia tienen un efecto hipoglucemiante significativo sin efectos adversos que aporta el mecanismo de acción sobre estimulando la secreción de insulina. Los resultados respaldan el hecho de que la extracción del principio activo de C. obtusifolia tienen un gran potencial para desarrollar un fármaco que mantenga los niveles normales de glucosa. [10]

Andrade-Cetto y Heinrich declararon que el posible mecanismo de la actividad de la planta es mediante la reducción en la producción de glucosa por la inhibición de Gl-6-P por ácido clorogénico, por lo que, puede atacar simultáneamente la gluconeogénesis y la glucogenólisis. [10]

En el Área de Yucatán Tradicionalmente, las hojas secas $(15 \mathrm{~g})$ se hierven en agua $(500 \mathrm{ml})$, y la infusión resultante se enfría en la olla antes de ser filtrado y bebido como "agua de uso" durante el transcurso de un día.

Podemos concluir que $\mathrm{C}$. obtusifolia tienen un efecto hipoglucemiante significativo sin efectos adversos $y$ aporta un efecto positivo en el mecanismo de acción en la secreción de insulina. Los resultados respaldan el hecho de que los extractos de $\mathrm{C}$. obtusifolia tienen un gran potencial para desarrollar un fármaco que permita mantener las concentraciones normales de glucosa mediante la extracción del principio activo. 


\section{GUAZUMA ULMIFOLIA}

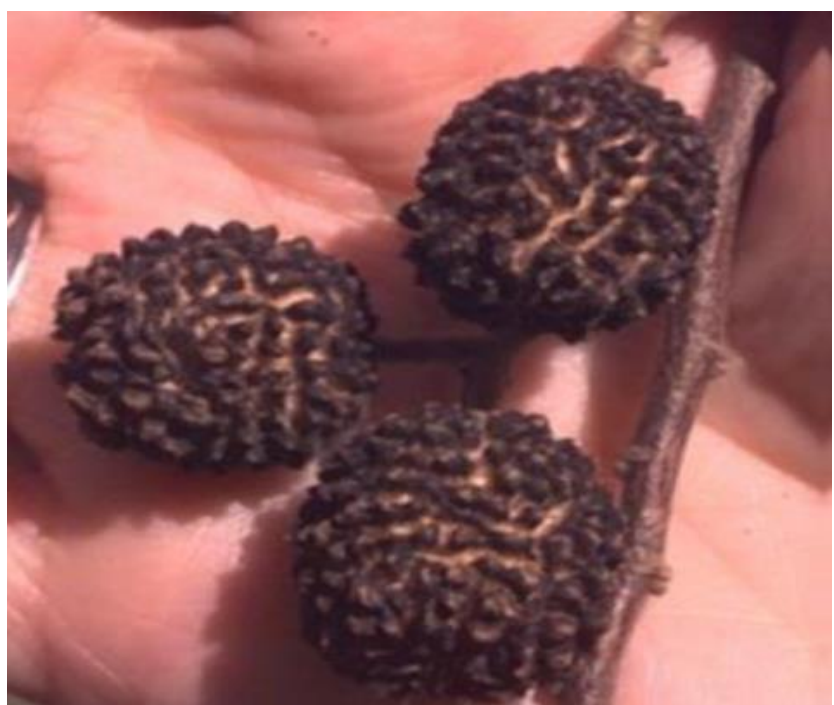

Figura 3. Nombre común: Palo de Guácima o palo cuchara. Nombre científico: Guazuma ulmifolia

Nombre en Tepehua: Aqaixta. Orden: Malvales Familia: Malvaceae Género: Guazuma

Uso medicinal: Es un gran antioxidante, ayuda a mantener normal los niveles de colesterol, las hojas suelen usarse para tratar la fiebre. También es usada para tratar normal los niveles de glucosa, tener una buena circulación y ayuda a acelerar el metabolismo, se toman infusiones de este fruto como agua de tiempo.

La diabetes mellitus tipo 2 (T2-D) es la enfermedad metabólica más común en todo el mundo. En México, T2$D$ es la primera causa de muerte en mujeres y el segundo en hombres. [11]

Además de la insulina, los medicamentos actualmente utilizados para el control de la diabetes mellitus tipo 2, incluyen derivados de sulfonilureas que estimulan la liberación de insulina por el páncreas, biguanidas que regulan la absorción de la glucosa a nivel intestinal y tiazolidinedionas (TZD) que restauran la respuesta a la insulina en los tejidos diana. Sin embargo, todos estos agentes tienen efectos secundarios toxicológicos y no pueden revertir las concentraciones glucémicas a cifras normales. [11]

Aproximadamente 1200 plantas se utilizan en todo el mundo para el tratamiento empírico de la T2-D. Sin embargo, solo alrededor de 350 de ellos están documentados científicamente para presentar actividad hipoglucemiante. [12]

En México, 306 especies de plantas de 235 géneros y 93 familias están documentadas para el tratamiento empírico de T2-D. [13]

En estudios desarrollados en cultivos celulares, mediante el empleo de Guazuma ulmifolia, los resultados mostraron que G. ulmifolia, tiene propiedades hipoglucémicas, estimulando la captación de glucosa en los adipocitos, sensibles y resistentes a los efectos de la insulina. Este efecto, se observó sin afectar el desarrollo del tejido adiposo, no ocasionando efectos pro-adipogénicos o antiadipogénicos, sugieriendo que $\mathrm{G}$. ulmifolia podría ser muy útil en el tratamiento de T2-D. [14]

En este contexto, se sugiere que Guazuma ulmifolia podría tener efectos ante los mecanismos que regulan la glucosa. Respondiendo a la insulina de dos maneras diferentes: por amplificación clonal de adipocitos recién comprometidos y aumentando la captación de glucosa en adipocitos maduros. Por lo tanto, concluyeron que los extractos acuosos de G. ulmifolia modifican cualquiera de estas dos actividades dependientes de insulina. Se demostró que G. ulmifolia no induce ni bloquea al factor 3T3 durante la adipogénesis, una propiedad deseable para cualquier fármaco utilizado para el control de la diabetes. [14]

De la misma forma, las personas de origen Tepehuana usan comúnmente las bolas secas de este árbol, mediante la ingesta de una infusión. Preparan de 4 a 6 bolas en 1 litro de agua, la cual van consumiendo durante todo el día como agua de tiempo.

\section{SOLANUM NIGRUM}

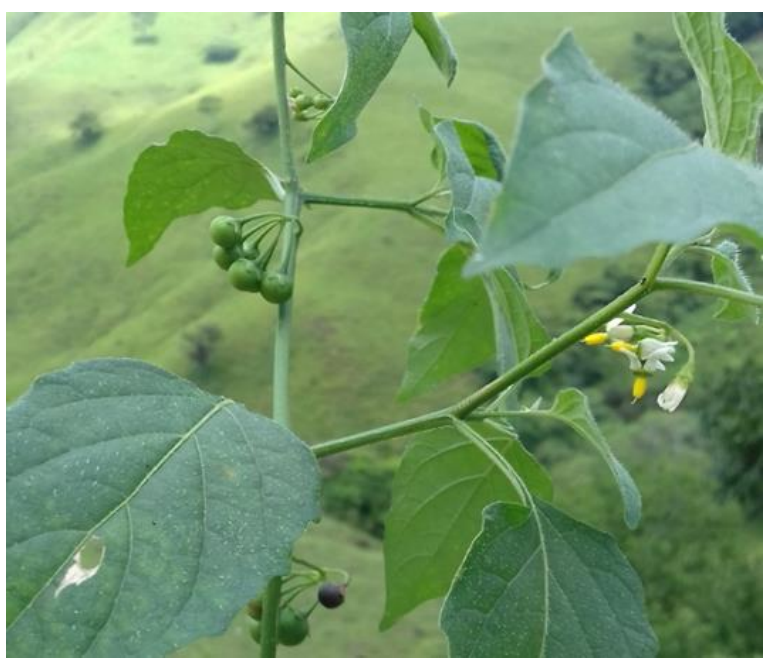

Figura 4. Nombre común: Hierba mora o tomatillo del diablo. Nombre científico: Solanum nigrum.

Nombre en Tepehua: Tampaks. Orden: Solanales.

Familia: Solanaceae Género: Solanum.

Uso medicinal: Se usa comúnmente para alergias dérmicas, su mayor uso es en pacientes con varicela, se toman baños de esta hoja refregada. También tiene propiedades que ayudan a tratar los cólicos menstruales, ayuda a aumentar el FG y mantener estables los niveles de glucosa. Asi mismo tiene un buen funcionamiento para tratar la tos. El fruto es un antioxidante y contiene toxinas que ayudan a tratar infecciones bucales 
Solanum nigrum (comúnmente llamado Makoi o hierba mora). Una ingesta dietética de $\mathrm{S}$. nigrum provee a nuestro cuerpo nutrientes que ofrecen protección contra numerosas enfermedades, los pueblos indígenas Tepehuanas la consumen como ensalada. Todas las partes de esta planta se utilizan en la medicina alternativa como remedio para tratar diversas enfermedades tales como: tos, fiebre, asma, enfermedades de la piel y problemas hepáticos.

La diabetes mellitus se ha convertido en un problema real de salud pública en países subdesarrollados. Este padecimiento es un desorden metabólico caracterizado por desarreglos en el metabolismo de carbohidratos, proteínas y grasas causado por la insuficiencia en la secreción y/o acción completa o relativa de insulina. Esto se debe a defectos en la respuesta secretoria de insulina. Resultando en una inadecuada captación de la glucosa deteriorado, que es un rasgo característico de la diabetes mellitus, es decir, la resultante hiperglucemia.

Se realizaron estudios en un modelo animal de ratas albinas maduras, a las cuales se les indujo diabetes, mediante una dieta. Días posteriores a la inducción de diabetes se les administró el extracto de $S$. nigrum, donde se observó una disminución de las concentraciones de glucosa después de 6hrs de su administración. [15] Los extractos de plantas medicinales han sido efectivos hipoglucemiantes y pueden involucrar a uno o más compuestos activos responsables de reducir la glucosa en sangre. El extracto de esta planta reveló la presencia de alcaloides y solaninas que pueden ser responsables de los efectos hipoglucémicos observados en estas fracciones, posiblemente estimulando la liberación de insulina desde las células beta pancreáticas. [15]

Otra investigación realizada por el Dr. Poongothai, en donde se indujo diabetes a ratas con alloxan, produciéndose un aumento significativo en las concentraciones de colesterol total $56.39 \%$, triglicéridos $87.79 \%$, LDL $85.77 \%$ y VLDL $87.81 \%$ mientras que los niveles de HDL disminuyeron en un $31.50 \%$ en el control diabético de ratas no tratadas en comparación con las normales. Después de la administración de $\mathrm{S}$. nigrum a concentraciones de $200,400 \mathrm{mg} / \mathrm{kg} . b . w$ disminuyeron los niveles de lípidos séricos a casi lo normal, provocando una significativa disminución del colesterol total de 16,97\%, $27,01 \%$, TG 36,03\%, 38,30\%, LDL 4.62\%, 30.03\%, VLDL $34.17 \%$ y $38.31 \%$. Por lo tanto, en este estudio se demostró que el grupo de ratas tratadas con extracto acuoso de hoja redujeron significativamente las concentraciones de glucosa en sangre en comparación con el grupo control diabético. [16]

En forma paralela, demostraron que los extractos de hojas acuosas de S. nigrum y Musa paradisiaca tienen importantes actividades hipoglucemiantes y antilipémicas en ratas diabéticas inducidas por aloxan, ya que se observó una mejora en parámetros como peso corporal, glucosa en sangre y perfil de lípidos, por lo tanto, podrían ser valiosos activos en el tratamiento de la diabetes. [16] De acuerdo a las investigaciones realizadas por Sugunabai y colaboradores, se encontró que después del tratamiento con extracto de $\mathrm{S}$. nigrum se disminuyeron los niveles de glucosa y los niveles de la hemoglobina glicosilada de $10.8 \%$ a $3.1 \%$ después del tratamiento con extracto acuoso de $\mathrm{S}$. nigrum, durante un tratamiento de 30 días, $16.8 \%$ a $10.3 \%$ durante 60 días y $18.9 \%$ a $12.3 \%$ por 90 días de tratamiento. De forma paralela hubo una disminución en el colesterol total de $6.6 \%$ en 30 días, $8.25 \%$ en 60 días y $10.3 \%$ en 90 días de tratamiento en comparación del inicio del proceso. Los niveles de triglicéridos mostraron también una disminución en su concentración por $9.9 \%$ en 30 días de tratamiento, $15.1 \%$ en 60 días y $16.5 \%$ en 90 días de tratamiento. Los niveles de LDL se redujeron a $6.6 \%$ en 30 días de tratamiento, $7.6 \%$ en 60 días, $8.0 \%$ en 90 días en comparación al inicio del tratamiento. No hubo modificación en las concentraciones de HDL durante el período de tratamiento. [17]

\section{CONCLUSIÓN:}

En este estudio se desarrolló un trabajo de campo en la comunidad indígena Tepehua, de Tierra Colorada, Tlachichilco, Veracruz. El siguiente paso, será realizar las extracciones de los diferentes principios activos de las plantas descritas anteriormente y de otras que no son referidas en este manuscrito. Posteriormente, se caracterizarán y emplearán en cultivos celulares y modelos animales en roedores, con el fin de evaluar sus efectos farmacológicos y toxicológicos. De esta manera podremos medir las reacciones en animales de prueba con inducción hacia diabetes mellitus, y usar los activos extraídos de las plantas como posibles fármacos, para finalmente ponerlo en práctica con pacientes que padezcan diabetes mellitus de tipo II, hipercolesterolemia e hipertensión arterial.

\section{Referencias}

[01] Garcia-Lujan C, Pérez Hernández BE, Martinez-Romero A, Castro Barraza F. Uso de plantas medicinales y suplementos dieteticos para el control glucemico de la diabetes. Revista Chapingo Serie Zonas Aridas. 2009:8;229-239.

[02] Manuel Ramiro H, J. H. El internista, Medicina interna para internistas. 12va edición. México DF: McGraw-Hill interamericana editores, S.A. de C.V. 2003: pp29.

[03] Beyra A, León MC, Iglesias E. Estudios etnobotánicos sobre plantas medicinales en la provincia de Camagüey (Cuba). Anales del Jardín Botánico de Madrid 2004:61;185-204. 
[04] Jung, M. Park M, Lee HC, Kang YH, Kang ES, Kim SK. Antidiabetic Agents from Medicinal Plants. Current Medicinal Chemistry. 2006:13;1203-1208.

[05] Kumar-Singh S, Kumar-Rai P, Jaiswal D, Watal G. Evidence-based Critical Evaluation of Glycemic Potential of Cynodon dactylon 2007:5;415-420.

[06] Samuelsen AB. The traditional uses, chemical constituents and biological activities of Plantago major. Journal of Ethnopatology 2000:71;1- 21 .

[07] Ceballos SJ, Perea MC. Plantas medicinales utilizadas por la comunidad indígena de Quilmes (Tucumán, Argentina). Boletín Latinoamericano y del Caribe de Plantas Medicinales y Aromáticas. 2014:13;47-68.

[08] Revilla-Monsalve MC, Andrade-Cetto A, Palomino-Garibay MA Wiedenfeld H, Islas-Andrade S. Hypoglycemic effect of Cecropia obtusifolia Bertol aqueous extracts on type 2 diabetic patients. Journal of ethnopharmacology. 2007:111; 636-640.

[09] Andrade-Cetto A, Vazquez RC. Gluconeogenesis inhibition and phytochemical composition of two Cecropia. Journal of ethnopharmacobiology. 2010:130;93-97.

[10] Andrade-Cetto A, Wiedenfeld. Hypoglycemic effect of Cecropia obtusifolia on streptozotocin diabetic rats. Journal of ethnopharmacobiology. 2001:78;145-149.

[11] Aguilar-Salinas CA, Velazquez-Monrroy O, Gómez-Pérez FJ. Characteristics of patients with type 2 diabetes in México: Results from a large population-basade nationwide survey. Diabetes care 2003:26; 2021-2026.

[12] Hernández-Galicia E, Aguilar-Contreras A, Aguilar Santamaria L.... Studies on hypoglycemic activity of Mexican medicinal plants. Proceedings of the western pharmacology society, 2000:45;118-124.

[13] Andrade-Cetto A, Heinrrich M. Mexican plants with hypoglycaemic effect used in the treatmen of diabetes. Journal of ethnopharmacobiology. 2005:99;325-348.

[14] Alonso-Castro AJ, Salazar-Olivo LA. The anti-diabetic properties of Guazuma ulmifolia Lam are mediatedby the stimulation of glucose uptake in normal and diabetic adipocytes without inducing adipogenesis. Journal of ethnopharmacology. 2008:118;252-256.

[15] Nazoora-Saleh A, Kusum S, Md-Iqbal K, Sapna R. Protective effect of ethanolic extract of solanum nigrum on the bloodsugar of albino rats. International journal of pharmaceutical sciences and research, 2010:1 97-99.

[16] Poongothai K, Ahmed KSZ, Ponmurugan P, Jayanthi M. Assessment of antidiabetic and antihyperlipidemic potential of Solanum nigrum and Musaparadisiaca in alloxan induced diabetic rats. Journal of Pharmacy Research. 2010:3;2203-2205.

[17] Sugunabai J, Jayaraj M, Karpagam T, Varalakshmi B. Antidiabetic efficiency of moringa oleifera and solanum nigrum. International Journal of Pharmacy and Pharmaceutical Sciences. 2014:6;40-42. 